techniques. Contrastingly, the final remaining third demonstrates five impressive clinical cases, presented in a similar meticulously detailed format [again without detailed explanation]. Readers of this book will also note that the science of occlusion or the details of aesthetics are only hinted at without being explored. This isn't the definitive stand-alone text on wax up. This is Paulo Kano presenting his technique as an art form. In that sense it fulfils its purpose, and would be well appreciated by students, technicians and clinicians alike.

\section{QDT 2011: QUINTESSENCE OF DENTAL TECHNOLOGY}

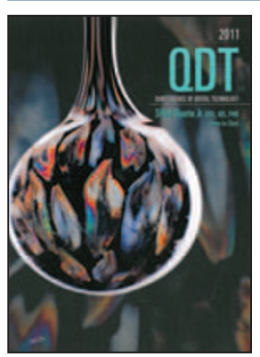

S. Duarte

UK: Quintessence

price $€ 60.00$; pp 200

ISBN 9780867155143

This most recent volume of Quintessence of dental technology is definitely another welcome addition to the series. In Sillas Duarte's third editorial he encourages dentists and dental technicians to take a 'calculated risk', giving examples of those who left their comfort zone and adopted newer techniques and materials to achieve successful aesthetic results. The annual is stylishly presented and features 15 easy to read articles with contributions from many different international professionals involved in cosmetic dentistry. All the articles are beautifully presented and contain a good variety of case reports, tables and a superb array of photographs.

Each of the articles is unique and covers the different aspects of cosmetic dentistry such as the ultraconservative approach to indirect restorations and optimal colour control and layering for all ceramic restorations. The cases presented are all very challenging clinical scenarios and the treatment plans are described and explained in great depth with annotated pre-, intra- and post-operative photographs and radiographs.
Although the treatment plans in these cases are all very complex the contributors all emphasise the involvement of the patient from the very early treatment planning stages through to the finished result. In addition to this the article on 'Excellence in dental aesthetics: a multidisciplinary challenge' emphasises the importance of teamwork in dentistry as the article describes how a well organised team of orthodontist, periodontists, implantologist and prosthodontist and dental technician all worked together to achieve a successful outcome.

By amalgamating the basic and complex aspects of clinical dentistry this book lends itself to both the enthusiastic dental student who wishes to see how boundaries of dentistry can be expanded to the experienced dental professional who wishes to enhance their practice and keep up to date with the new developments in cosmetic dentistry. A reader of any level would find this an enjoyable read and even if they do not wish to take a "calculated risk' will pick up a few tips to help them in their daily practice. Dental technicians too would find this book of interest with articles on the platinum foil technique and internal staining of indirect restorations.

I would strongly recommend this book as it enthusiastically demonstrates how stunning clinical results can be achieved and with this annual Sillas Duarte's own 'calculated risk' has certainly paid off.

P. Mooney 